\title{
Ectopic cervical thymus as a rare cause of pediatric neck mass: the role of ultrasound and MRI in the diagnosis. Case report.
}

\author{
Alper Ozel ${ }^{1}$, Pınar Ozdemir Akdur ${ }^{1}$, Irfan Celebi ${ }^{1}$, Rabia Karasu ${ }^{1}$, Banu Yılmaz ${ }^{2}$, Muzaffer \\ Basak $^{1}$
}

${ }^{1}$ Radiology Department, ${ }^{2}$ Pathology Department, Sisli Hamidiye Etfal Research and Training Hospital, Sisli, Istanbul, Turkey

Abstract

Ectopic cervical thymus (ECT) is an uncommon cause for cervical mass in the pediatric age group. Ultrasound and magnetic resonance imaging findings of the mass (location along the thymic descent pathway along the thymopharyngeal tract and the identical echostructure and signal intensities to the native thymus in the superior mediastinum) would lead to the diagnosis. The diagnosis is confirmed by fine needle aspiration biopsy or histopathology after resection. The management of ECT is a conservative follow up, except in symptomatic cases with tracheal compression and histologically confirmed neoplasia where surgery is indicated. We present the case of ECT presenting as a left upper neck mass in a 12 year old girl.

Keywords: thymus, ectopic, ultrasonography, MRI

\section{Introduction}

Neck masses are fairly common during infancy and childhood. In the differential diagnosis list, there are a variety of causes including thyroglossal duct cyst, branchial cleft cyst, aberran thyroid, tumors arising from the thyroid and parathyroid, cervical lymphadenopathies, vascular tumors, benign and malignant tumors of the neck. Ectopic cervical tyhmus (ECT) is a rare cause of neck masses and can pose a diagnostic dilemma. Here, we report an ECT presenting as a left upper neck mass in a 12 year old girl, diagnosed with ultrasound and MRI.

\section{Case Report}

A 12 year old girl presented to her pediatrician with a palpable nontender nodule on her left side of the neck. Clinical examination revealed an afebrile child with a

Received 22.09.2014 Accepted 15.10.2014

Med Ultrason

2015, Vol. 17, No 2, 248-251

Corresponding author: Alper Ozel, M.D.

Aytekin Kotil Cad. No:18 D:20

34360 - Sisli, Istanbul / Turkey

Mobile: +90 5324311834

E-mail: dralperozel@gmail.com palpable soft mobile nodule in the submandibular area below the mandibular angle. The laboratory tests results were normal. The patient was then referred to radiology department for ultrasound evalulation of the palpable lump. The ultrasound examination was performed with a 5-14 MHz high frequency linear array transducer (Siemens S2000, Siemens Healthcare, Erlangen, Germany) with the patient's neck slightly extended. A $37 \times 20 \times 10.5$ $\mathrm{mm}$ sized well defined, hypoechoic lesion with punctate and linear echogenic foci was detected sonographically laterally to the left submandibular gland. The lesion was molding over the carotid vessels without invading or displacing them. It had a fingerlike projection insinuating between the left common carotid artery and internal jugular vein (fig 1a, b). In a suprasternal approach, it could be clearly seen that the lesion had the same echostructure with the native thymus (fig 1c). The initial impression for the diagnosis of the cervical mass was ECT. To verify the diagnosis and to demonstrate any possible additional ectopic thymic tissue along both sides of the neck, MRI (Siemens Avanto, Siemens, Erlangen, Germany) was performed. The mass adjacent to the submandibular gland was isointense to muscle on $\mathrm{T} 1$ and hyperintense on $\mathrm{T} 2$ weighted sequences. After an intravenous Gadolinium injection, it showed moderate enhancement (fig 2). The 


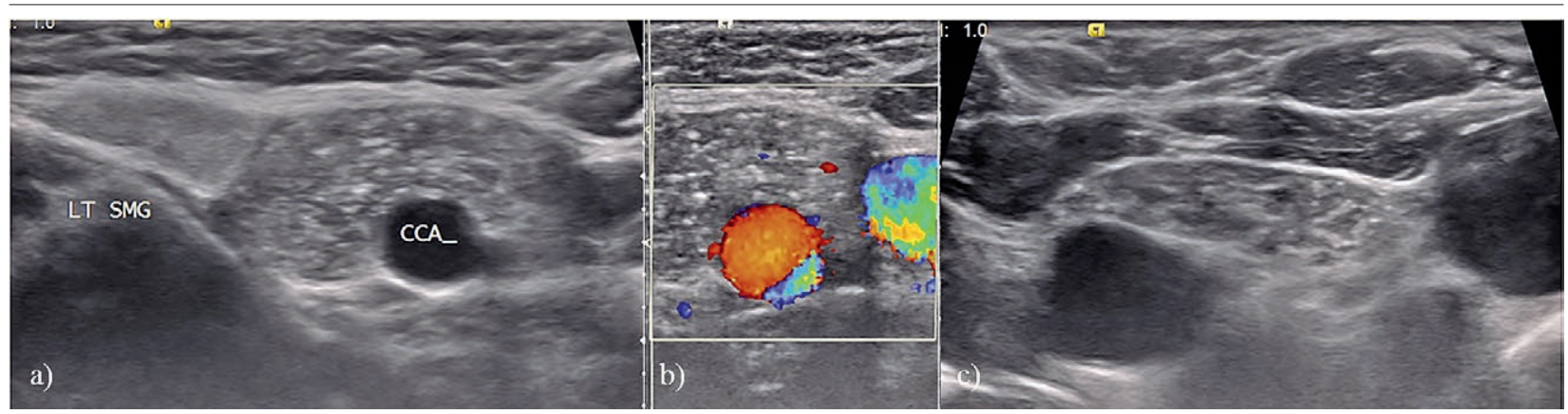

Fig 1. a) Left transverse sonogram of the neck. A hypoechoic heterogenous lesion with punctate echogenic foci and hyperechoic linear striation is seen laterally to the submandibular gland; b) color Doppler examination of the mass. The mass, which is almost avascular, is insinuating between the carotid artery and internal jugular vein. There is no mass effect on the vascular structures; c) transverse caudal suprasternal sonographic approach showing the native thymic tissue in the superior mediastinum.

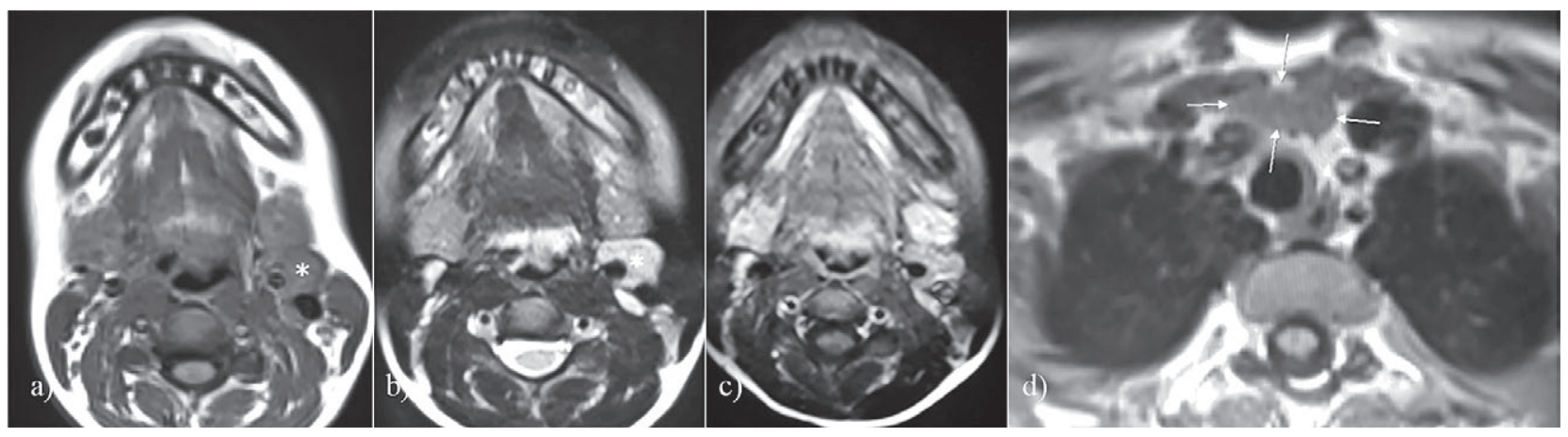

Fig 2. a) T1 weighted axial MR image demonstrates a well defined isointense mass (marked with white *) to muscle between left submandibular gland and major vascular structures; b) T2 weighted axial MR image demonstrates a well defined hyperintense mass (marked with white*) with fingerlike projection insinuating between left common carotid artery and internal jugular vein; c) Axial post Gadolinium T1 weighted image demonstrates moderate enhancement of the mass; d) Axial T1 weighted image at the level of superior mediastinum demonstrates native thymic tissue (delineated with white arrows) with signal intensity identical to ECT.

signal intensity characteristic and enhancement after contrast was the same with the native thymus in the superior mediastinum.

The fine needle aspiration biopsy (FNAB) performed under ultrasound guidance with 27 gauge needle demonstrated groups of small lymphocytes. Ectopic cervical thymus was diagnosed in accordance with the imaging and cytopathological findings. There was no complication after the FNAB. The decision for follow up was considered.

\section{Discussions}

ECT results from migrational defects during thymic embryogenesis. The thymus is a paired organ that is recognizable early in the 6th week of fetal life. The thymus is embryologically derived from the ventral wings of the third pharyngeal pouch on each side. During the eighth week, each thymic primordium elongates caudally and medially and forms the thymopharyngeal duct. As the primordial thymus descents into its ultimate position into the anterior superior mediastinum, the upper end of the thymus is drawn out and eventually disappears. The remaining endodermal epithelium degenerates into the concentric thymic corpuscules of Hassall. By the third embryological month, the thymus develops a cortex and a medulla $[1,2]$. Ectopic thymic tissue can be found anywhere along the thymopharyngeal duct.

ECT has been described infrequently in pediatric literature. The theories explaining the ECT include hyperplasia of undescendent or sequestrated thymic remnants and persistance of thymopharyngeal duct. It can present clinically as a cervical mass that can be solid, cystic or mixed. It is believed that cystic ECT occurs as a result of cystic degeneration of Hassall's corpuscles or a glandular duct epithelium in cervical thymic remnants $[2,3]$.

$\mathrm{ECT}$ is an an uncommon cause of pediatric neck mass and usually detected incidentally. However, the prevalence of ECT with the clinically occult cases is higher than as thought according to a study reporting the occurrence of ECT in $21 \%$ to $31 \%$ of children at autopsy [4]. The differential diagnosis of a neck mass in the pediat- 
ric age group is extensive and includes more common causes such as thyroglossal duct cyst, branchial cleft cyst, sternocleidomastoid fibroma, tumors arising from thyroid and parathyroid, cervical lymphadenopaties, vascular tumors, benign and malignant tumors [5].

Ultrasound is the most direct and practical modality for the diagnosis of ECT. The increased resolution with the improvement of transducer technology, lack of ionizing radiation and the relatively short examination time makes ultrasound as the initial advantageous modality in imaging of the neck of the pediatric population. Familiarity with the ultrasound appearance is useful in the diagnosis of ECT and preventing unnecessary surgeries $[6,7]$. Sonographic appearance of the normal thymus is described as a well defined homogenous, hypoechoic mass with multiple echogenic structures and internal echogenicities [8-13]. In an ultrasound-pathology correlation study of intrathyroid anatomy, the echogenic linear structures within the thymus represent connective tissue septa and blood vessels in the septa [9]. This characteristic echostructure of a cervical mass is a clue to the diagnosis of ECT.

MRI with its superior soft tissue contrast provides significant information about the nature of the cervical mass. On MRI, ectopic thymus is homogenous, isointense, or slightly hyperintense compared with muscle on T1 weighted images, and hyperintense on T2 weighted images. The signal intensities of ECT is strikingly similar to these of the normal located thymus. MRI has been shown to be an excellent modality in the visualization of any remaining thymic tissue between the predominant ectopic thymus and mediastinal thymus anywhere along the thymopharyngeal duct [14]. The morphological features of ECT are common on US and MRI. These masses often have an angulated configuration and mould over adjacent structures without invading and displacing them.

In the literature, in nearly all children with ECT, the lesion was diagnosed with excision. In recent literature however, there has been a diagnostic algorithm shift from surgery or biopsy to radiological imaging findings only $[15,16]$. Although malignant transformation in ECT has been reported in rare cases, there is no convincing evidence of it. In 1954, Pirkey reported a case of thymoma arising in a ECT, which was of epithelial origin and not metastatic [17]. In a review of 32 cases of ECT compiled by Lewis, malignant cells have been reported in a tracheal mass in a 58 year-old female [18]. Additionally, there are no data indicating that the incidence of malignant transformation of ECT is any greater than in normal thymic tissue. According to this knowledge and to only one published thymoma case in childhood with no metastasis, and due to the natural involution process of ectopic thymus that begins at puberty, it is acceptable not to take a biopsy or surgically remove ectopic thymic tissue in the neck.

Han et al claimed that it is possible to diagnose ECT with US alone and there is no need for surgical excision [6]. In their case report and literature review, Zielke et al conclude that ECT can be confirmed with US and the overall geographic distribution can be nicely demonstrated with MR imaging. Their suggestion for an asymptomatic cervical mass in a child incidentally found along the path of thymic descent with US and MR imaging features of ECT, is that there is no need for biopsy or surgical removal [11].

In conclusion, ectopic cervical thymus is a rare cause of pediatric neck mass. Ultrasound and MRI, with the imaging findings of the mass along the thymopharyngeal tract similar to the native thymus, and fine needle aspiration allow the diagnosis to be made. ECT can be followed up conservatively, except in symptomatic cases with tracheal compression, histologically confirmed neoplasia, and in patients with inconclusive FNA diagnosis.

\section{References}

1. Tovi F, Mares AJ. The aberran cervical thymus. Embryology, pathology, and clinical implications. Am J Surg 1978; 136: 631-637.

2. Boyd J, Templer J, Havey A, Walls J, Decker J. Persistent thymopharyngeal duct cyst. Otolaryngol Head Neck Surg 1993; 109: 135-139.

3. Khariwala SS, Nicollas R, Triglia JM, et al. Cervical presentation of thymic anomalies in children. Int J Pediatr Otolaryngol 2004; 68: 909-914.

4. Bieger RC, McAdams AJ. Thymic cysts. Arch Pathol 1966; 82: 535-541.

5. Millman B, Pransky S, Castillo J 3rd, Zipfel TE, Wood WE. Cervical thymic anomalies. Int J Pediatr Otorhinolaryngol 1999; 47: 29-39.

6. Han BK, Yoon HK, Suh YL. Thymic Ultrasound. II. Diagnosis of aberran cervical thymus. Pediatr Radiol 2001; 31: 480-487.

7. Koumanidou C, Vakaki M, Theophanopoulou M, et al. Aberrant thymus in infants: sonographic evaluation. Pediatr Radiol 1998; 28: 987-989.

8. Han BK, Babcock DS, Oestreich AE. Normal thymus in infancy: sonographic characteristics. Radiology 1989; 170: 471-474.

9. Han BK, Suh YL, Yoon HK. Thymic ultrasound. I. Intrathymic anatomy in infants. Pediatr Radiol 2001; 31: 474479.

10. Adam EJ, Ingotus PI. Sonography of thymus in healthy children: frequency of visualisation, size and appearance. AJR Am J Roentgenol 1993; 161: 153-155. 
11. Zielke AM, Swischuk LE, Hernandez JA. Ectopic cervical thymic tissue: can imaging obviate biopsy and surgical removal? Pediatr Radiol 2007; 37: 1174-1177.

12. Fitoz S, Atasoy C, Turkoz E, Gumus D, Erden I, Akyar S. Sonographic findings in ectopic cervical thymus in an infant. J Clin Ultrasound 2001; 29: 523-526.

13. Herman TE, Siegel MJ. Cervical ectopic thymus. J Perinatol 2009; 29: 173-174.

14. Slovis TL, Meza M, Kuhn JP. Aberrant thymus - MR assessment. Pediatr Radiol 1992; 22: 490-492.
15. Schloegel LJ, Gottschall JA. Ectopic cervical thymus: Is empiric surgical excision necessary? Int J Pediatr Otolaryngol 2009; 73: 475-479.

16. Song I, Yoo SY, Kim JH, Hong E, Yoon HK. Aberrant cervical thymus: imaging and clinical findings in 13 children. Clin Radiol 2011; 66: 38-42.

17. Pirkey W. Epithelioma of undescended thymus in a child. Arch Otolaryngol 1954; 59: 237-240.

18. Lewis MR. Persistence of the thymus in the cervical area. J Pediatr 1962; 61: 887-893. 